\title{
Desenvolvimento e caracterização de doce misto cremoso de coco verde e abacaxi
}

\author{
Development and characterization of creamy mixed sweet of green coconut and pineapple \\ Elaboración y caracterización de dulces mixtos cremosos de coco verde y piña
}

Tatiana Zanella Rodrigues

ORCID: https://orcid.org/ 0000-0002-1623-3314

Universidade Federal da Paraíba, Brasil

E-mail: tzrodrigues@hotmail.com

Maria Gorette de Queiroz Oliveira

ORCID: https://orcid.org/0000-0001-7023-3027

Universidade Federal da Paraíba, Brasil

E-mail: goretteq@hotmail.com

Taísa Vieira Fernandes

ORCID: https://orcid.org/ 0000-0003-1870-9504 Universidade Federal da Paraíba, Brasil

E-mail: taisavieira8@gmail.com

Noádia Priscila Araújo Rodrigues

ORCID: https://orcid.org/0000-0003-2721-5768 Universidade Federal da Paraíba, Brasil E-mail: noadia_priscila@hotmail.com

Patrícia Pinheiro Fernandes Vieira

ORCID: https://orcid.org/0000-0003-0392-3698 Universidade Federal da Paraíba, Brasil E-mail: patriciaprs@gmail.com

Ingrid Conceição Dantas Guerra

ORCID: https://orcid.org/0000-0002-8729-646X Universidade Federal da Paraíba, Brasil E-mail: ingridcdantas@hotmail.com

\begin{abstract}
Resumo
O albúmen sólido do coco verde é um coproduto da indústria da água de coco que possui elevada qualidade nutricional, mas é amplamente desperdiçado no Brasil. O objetivo deste estudo foi avaliar a viabilidade de utilização deste coproduto na produção de doces mistos de coco e abacaxi e realizar a caracterização dos mesmos. Foram elaboradas três formulações (F1 4,8\% de abacaxi e de coco, F2 coco verde 50\%, abacaxi 24,25\% e F3 coco verde $59,7 \%$ e abacaxi $10 \%$ ) e realizadas análises microbiológicas, físico-químicas e sensoriais (aceitação e intenção de compra).Os resultados revelaram que todas as formulações se apresentaram seguras para consumo humano tendo as contagens abaixo do limite de deteç̧ão $(<100 \mathrm{Log}$ UFC $/ \mathrm{mL})$ para Enterobactérias e Bolores e leveduras e a ausência de Salmonella spp. Todas as formulações se apresentaram com $\mathrm{pH}$ ácido, sendo a acidez maior $(\mathrm{p}<0,05)$ nas formulações com maior quantidade de abacaxi. Na avaliação sensorial, todas as formulações foram aceitas tendo notas acima do ponto neutro, mas a formulação F1 recebeu conceitos maiores e maior percentual de intenção de compra ao passo que a F3 obteve o maior percentual de rejeição na intenção de compra. A utilização do albúmen sólido de coco verde apresentou-se como alternativa para utilização na produção de doce de coco e abacaxi tendo resultado em produtos microbiologicamente seguros, com características físico-químicas dentro do previsto pela legislação e bem aceitos sensorialmente sendo, portanto, uma alternativa para combater o desperdício dessa matéria-prima no Brasil.
\end{abstract}

Palavras-chave: Desperdício; Resíduo alimentar; Coco verde.

\begin{abstract}
The solid albumen of the green coconut is a co-product of the coconut water industry that has high nutritional quality, but is widely wasted in Brazil. The aim of this study was to evaluate of using this co-product in the production of mixed coconut and pineapple sweet and to characterize them. Three formulations were prepared (F1 4.8\% pineapple and coconut, F2 green coconut 50\%, pineapple $24.25 \%$ and F3 green coconut $59.7 \%$ and pineapple $10 \%$ ) and microbiological, physical-chemical and sensory analyzes were performed (acceptance and purchase intention). The results revealed that all formulations were safe for human consumption with counts below the detection limit $(<100$ Log UFC / mL) for Enterobacteria and Molds and yeasts and the absence of Salmonella spp. All formulations were presented with acidic $\mathrm{pH}$, with the highest acidity $(\mathrm{p}<0.05)$ in the formulations with the highest amount of pineapple. In the sensory evaluation, all formulations were accepted with scores above the neutral point, but the F1 formulation received higher concepts and a higher percentage of purchase intention whereas F3 obtained the highest percentage of rejection in purchase intention. The use of solid green coconut albumen presented itself as an alternative for use in the
\end{abstract}


production of coconut and pineapple candy, resulting in microbiologically safe products, with physico-chemical characteristics within the scope of the legislation and well accepted by the senses, being, therefore, an alternative to combat the waste of this raw material in Brazil.

Keywords: Waste; Food residue; Green coconut.

\section{Resumen}

La albúmina sólida del coco verde es un coproducto de la industria del agua de coco que tiene una alta calidad nutricional, pero se desperdicia ampliamente en Brasil. El objetivo de este estudio fue evaluar el uso de este coproducto en la producción de dulces mixtos de coco y piña y caracterizarlos. Se prepararon tres formulaciones (F1 $4.8 \%$ piña y coco, F2 coco verde $50 \%$, piña $24.25 \%$ y F3 coco verde $59.7 \%$ y piña $10 \%$ ) y se realizaron análisis microbiológicos, físico-químicos y sensoriales (aceptación e intención de compra). Los resultados revelaron que todas las formulaciones eran seguras para el consumo humano con recuentos por debajo del límite de detección $(<100$ Log UFC / mL) para enterobacterias y mohos y levaduras y la ausencia de Salmonella spp. Todas las formulaciones se presentaron con $\mathrm{pH}$ ácido, con la mayor acidez $(\mathrm{p}<0.05)$ en las formulaciones con mayor cantidad de piña. En la evaluación sensorial se aceptaron todas las formulaciones con puntajes por encima del punto neutro, pero la formulación F1 recibió conceptos más altos y un mayor porcentaje de intención de compra mientras que F3 obtuvo el mayor porcentaje de rechazo en la intención de compra. El uso de albúmina de coco verde sólido se presentó como una alternativa para su uso en la elaboración de dulces de coco y piña, dando como resultado productos microbiológicamente seguros, con características físico-químicas dentro del alcance de la legislación y bien aceptados por los sentidos, siendo, por tanto, , una alternativa para combatir el desperdicio de esta materia prima en Brasil.

Palabras clave: Residuos; Residuos de alimentos; Coco verde.

\section{Introdução}

O coqueiro é uma importante cultura perene, pois consegue se desenvolver em quase todas as regiões tropicais do globo (Dias, 2019). Possui origem asiática (Malásia, Filipinas e Indonésia) e foi introduzido no Brasil em 1553, no estado da Bahia, se disseminando rapidamente pela costa litorânea graças a adaptação as condições edafoclimáticas (Lima et al., 2015).

O Brasil é o quinto produtor mundial de coco, com 1,7\% da área cultivada e 3,8\% da produção mundial, sendo que a maior parte da produção está na região Nordeste do país (Brainer, 2018). A localização dessa região em área de baixada litorânea e tabuleiros costeiros favorece o plantio e torna a região responsável por aproximadamente $70 \%$ da produção do coco brasileiro (Martins \& Jesus Júnior, 2014).

A espécie Cocos nucífera L. pertence à família Arecaceae e possui algumas variedades a saber: $C$. nucifera var. typica (coqueiro gigante) e $C$. nucifera var. nana (coqueiro anão), além da híbrida, resultante do cruzamento entre essas duas variedades. O fruto é caracterizado como uma drupa fibrosa e é formado pelo epicarpo ou exocarpo (responsável pela cor verde ou amarela do coco), mesocarpo (parte fibrosa) endocarpo (camada rígida) endosperma sólido ou albúmen sólido (conhecido por polpa) e endosperma líquido ou albúmen líquido (conhecido por "água de coco") (Vysach et al., 2014). A variedade gigante é importante economicamente pela utilização do albúmen sólido na produção de alimentos e indústria cosmética e a variedade anã é amplamente produzida para comercialização do albúmen líquido (água de coco) (Benassi et al., 2013).

Sendo um país tropical e com uma ampla faixa litorânea o consumo de água de coco está em pleno crescimento e já atinge a marca de 350 milhões de litros por ano tanto de forma fresca como industrializada (resfriada e/ou pasteurizada). A água de coco além de ser apreciada por seu sabor e frescor, é considerada um excelente isotônico natural, por isso também é consumido por suas qualidades nutricionais (Santana et al., 2011).

Apesar do benefício à saúde, o aumento do consumo de água de coco leva também ao aumento na quantidade de resíduos sólidos que são gerados da comercialização do mesmo. Aproximadamente 23 milhões de toneladas de coco são destinados à extração da água, e a Paraíba está entre os 10 estados que mais geram resíduo de coco verde no Brasil (Instituto Brasileiro de Geografia e Estatística - IBGE, 2017). 
Junto ao resíduo do coco está o albúmen sólido (polpa de coco verde), que possui boas qualidades nutricionais, é rico em água, açúcares, proteínas e gorduras em sua composição e, portanto, tem um grande potencial econômico se utilizado na elaboração de novos produtos. No entanto, ainda é pouco utilizado sendo este coproduto descartado após a retirada da água (Teixeira et al., 2019).

Na literatura científica, são poucos os estudos que se direcionaram ao aproveitamento do albúmen sólido do coco verde, no entanto, os que foram realizados a exemplo de smothie (Teixeira et al., 2019), Iogurte (Luz et al., 2020); Biomassa (Machado et al., 2020) mostraram resultados promissores uma vez que os produtos se mostraram seguros quanto as características microbiológicas e de boa aceitação sensorial. Neste sentido, produtos que utilizem o albúmen sólido e que possuam potencial de utilização pela indústria a exemplo dos doces, mostram-se importantes economicamente e ambientalmente por propor um destino sustentável a este coproduto.

A legislação brasileira denomina doce em pasta o produto resultante do processamento adequado de partes comestíveis desintegradas de vegetais com açúcares, com ou sem adição de água, pectina, ajustador de pH e outros ingredientes, aditivos permitidos por estes padrões até uma consistência apropriada, sendo finalmente, acondicionados de forma a assegurar sua perfeita conservação (Brasil, 1978). Quanto a classificação os doces em pasta podem ser simples quando feitos com um único vegetal ou mistos quando feitos com mais de um e cremosos quando estão pastosos e não apresentam necessidade de corte (Brasil, 1978).

A produção de doces em pasta mistos mostra-se como uma alternativa interessante para utilização do albúmen sólido do coco verde, haja vista o açúcar atuar como conservante em uma matéria-prima que é rica em água. A possibilidade de usar o albúmen sólido do coco verde junto de outras frutas como o abacaxi também é promissora visto que podem promover diversificação de sabores além de proporcionar acidez de forma natural.

Face ao exposto, o objetivo deste estudo foi desenvolver doces em pasta mistos de coco verde e abacaxi e realizar a caracterização microbiológica, físico-química e sensorial das formulações.

\section{Metodologia}

Com base em seus objetivos esta pesquisa caracterizou-se como uma pesquisa explicativa. As pesquisas explicativas têm como preocupação central identificar os fatores que determinam ou que contribuem para a ocorrência dos fenômenos. Quanto ao procedimento técnico utilizado esta pesquisa classifica-se como experimental e quantitativa, que consiste essencialmente em determinar um objeto de estudo, selecionar as variáveis capazes de influenciá-lo e definir as formas de controle e de observação dos efeitos que a variável produz no objeto (Gil, 2002).

Para a obtenção do albúmen sólido (polpa) do coco verde foi feito contato com um vendedor ambulante no município de João Pessoa - Paraíba e as amostras foram coletadas no dia em que o vendedor abriu os cocos para encher as garrafas de polietileno para comercialização do albúmen líquido (água de coco). Imediatamente após a abertura, os cocos foram acondicionados em sacos estéreis e levados ao Laboratório de Cozinha Experimental pertencente ao Centro de Tecnologia e Desenvolvimento Regional (CTDR) da Universidade Federal da Paraíba (UFPB) para a retirada do albúmen sólido. Em ambiente laboratorial os cocos foram abertos na metade com auxílio de uma faca e a polpa foi cuidadosamente retirada com auxílio de uma colher. Os demais ingredientes utilizados (abacaxi, açúcar cristal e pectina) foram obtidos no comércio local do município de João Pessoa - PB.

Foram elaboradas três formulações de doce misto cremoso com diferentes proporções de albúmen sólido de polpa verde e abacaxi, seguindo as recomendações descritas por Torrezan (2015). As formulações estão expressas na Tabela 1. 
Tabela 1. Formulações de doces mistos de coco verde e abacaxi cremosos.

\begin{tabular}{ccccc}
\hline Ingredientes & & Formulações & F3 \\
\cline { 3 - 5 } \cline { 3 - 5 } & F1 & F2 & $59,7 \%$ \\
Coco verde & $34,85 \%$ & $50 \%$ & $10 \%$ \\
Abacaxi & $34,85 \%$ & $24,25 \%$ & $30 \%$ \\
Acúcar & $30 \%$ & $25,5 \%$ & $0,3 \%$ \\
Pectina & $0,3 \%$ & $0,25 \%$ & $0.3 \%$ \\
\hline
\end{tabular}

Fonte: Autores (2020).

Os abacaxis foram sanitizados em solução de hipoclorito de sódio (1 mL/100 mL por 15 minutos) em seguida foram descascados e os talos foram retirados. Tanto o abacaxi como a polpa de coco verde foram cortados em cubos, pesadas conforme as formulações, trituradas em multiprocessador de alimentos (Walita, São Paulo, Brasil) e levadas ao fogo em panelas de inox. Após fervura por 10 minutos adicionou-se metade da quantidade de açúcar e aqueceu-se por mais 10 minutos. Ao final, adicionou-se a pectina misturada ao restante do açúcar e aqueceu-se até as formulações atingirem o mínimo de $55^{\circ}$ Brix medido em refratômetro digital. Os doces foram transferidos para vidros estéreis e imediatamente resfriados a $85^{\circ} \mathrm{C}$, fechados e rotulados.

Para caracterizar os doces elaborados, as formulações foram submetidas a testes microbiológicos para detecção de enterobactérias, bolores e leveduras e pesquisa de Salmonella sp conforme recomendação da legislação brasileira para doces em pasta (BRASIL, 2019) e segundo metodologia descrita pela (APHA,2001). Para caracterização dos doces quanto aos parâmetros físico-químicos foram realizadas análises de umidade em estufa a $105^{\circ} \mathrm{C}$, o $\mathrm{pH}$ foi determinado usando pHmetro digital (DIGIMED, modelo pH 300M, São Paulo, Brazil), Acidez titulável foi determinada usando fenoftaleína como indicador em presença de $\mathrm{NaOH}$ a 0,1 N e os sólidos solúveis foram medidos em refratômetro digital (NOVA, WAY-2S, Piracicaba, São Paulo, Brasil) conforme metodologias descritas pela AOAC (2016).

As amostras também foram submetidas à análises sensoriais de aceitação e intenção de compra conforme metodologias propostas por Meilgaard et al., (2006) e Stone e Sidel (2004). A realização dos testes foi previamente aprovada pelo Comitê de Ética em pesquisa do Centro de Ciências da Saúde - CCS da UFPB e o painel foi composto de 60 painelistas não treinados composto de estudantes, funcionários e professores da UFPB.

Os testes sensoriais foram conduzidos nas dependências do Laboratório de Análise Sensorial CTDR/UFPB e realizados em cabines individuais com iluminação branca. Os painelistas receberam aproximandamente 40g de cada amostra, codificadas com números aleatórios de três dígitos para serem avaliadas monadicamente e foram orientados a provar biscoito água e sal e beber água entre as amostras para evitar sabores residuais entre as amostras.

Cada formulação foi avaliada quanto aos atributos de aparência, cor, odor, sabor, textura e aceitação global usando uma escala hedônica de nove pontos onde 1 = Desgostei muitíssimo, 5 = nem gosto/nem desgosto e $9=$ Gostei muitíssimo. Os painelistas também foram submetidos a um teste de intenção de compra, utilizando uma escala hedônica de cinco pontos onde 1 = certamente não compraria, 3 = talvez comprasse/talvez não comprasse e 5 = certamente compraria.

Todas as análises foram realizadas em triplicata e os resultados foram expressos como a média das três repetições. As análises estatísticas foram realizadas por meio de estatística descritiva (média e desvio padrão) e testes inferenciais (ANOVA seguida do teste de Tukey) para determinar diferenças estatisticamente significativas $(\mathrm{p} \leq 0,05)$ entre os tratamentos. Para as análises estatísticas, foi utilizado o software computacional Sigma Stat 2.03. 


\section{Resultados e Discussão}

Os resultados da avaliação microbiológica das formulações de doce misto cremoso de coco verde e abacaxi estão expressos na Tabela 1. Os resultados obtidos se apresentaram abaixo do limite de detecção estando todos dentro do preconizado pela legislação brasileira que traz como aceitável, 10 para enterobactérias, $10^{3}$ para bolores e leveduras e ausência de Salmonella sp (Brasil, 2019).

Tabela 1. Contagem de microrganismos e pesquisa de Salmonella sp. em doces mistos de coco verde e abacaxi cremosos.

\begin{tabular}{cccc}
\hline Variáveis & & Formulações & F3 \\
\cline { 2 - 4 } & F1* & F2 & $<100$ \\
$\begin{array}{c}\text { Enterobactérias (UFC/g) } \\
\text { Bolores e leveduras } \\
\text { (UFC/g) }\end{array}$ & $<100$ & $<100$ & $<100$ \\
$\begin{array}{c}\text { Salmonella } \text { sp. } \\
\text { (Presença/ausência) }\end{array}$ & $<100$ & $<100$ & Ausência \\
\hline
\end{tabular}

*F1(34,8\% de abacaxi e de coco) F2 (coco verde 50\%, abacaxi 24,25\%) e F3 (coco verde 59,7 e abacaxi 10\%). Fonte: Autores (2020).

A elevada qualidade microbiológica dos doces possivelmente estão relacionadas às boas práticas de fabricação, ao tratamento térmico e a baixa disponibilidade de água em virtude do açúcar adicionado. $\mathrm{O}$ tratamento térmico aliado ao açúcar atua como conservante, e unidos tornam-se barreiras ao crescimento microbiano. Outros estudos realizados com doces também obtiveram contagens abaaixo do limite de detecção assim como neste estudo (Silveira et al., 2020).

Os resultados da caracterização físico-química estão expressos na Tabela 2. Com exceção dos valores para sólidos solúveis, houve diferença estatística entre as formulações nos parâmetros avaliados $(\mathrm{p}<0,05)$.

Tabela 2. Escores médios e desvios-padrão das análises físico-químicas de doces mistos de coco verde e abacaxi cremosos.

\begin{tabular}{lccc}
\hline \multicolumn{1}{c}{ Variáveis } & \multicolumn{2}{c}{ Formulações } & F3 \\
\cline { 2 - 4 } & F1* & F2 & $32,89^{\mathrm{a}} \pm 0.67$ \\
Umidade $(\%)$ & $30,44^{\mathrm{b}} \pm 0.01$ & $30,51^{\mathrm{cb}} \pm 0.7$ & $6,02^{\mathrm{a}} \pm 0.05$ \\
$\mathrm{pH}$ & $5,01^{\mathrm{b}} \pm 0.03$ & $5,20^{\mathrm{c}} \pm 0.05$ & $0,48^{\mathrm{b}} \pm 0.01$ \\
Acidez $\left(\mathrm{mg} / 100 \mathrm{~g}^{-1}\right.$ de ácido cítrico) & $1,12^{\mathrm{a}} \pm 0.29$ & $0,64^{\mathrm{ab}} \pm 0.28$ & $65,3^{\mathrm{ab}} \pm 0.00$ \\
Sólidos Solúveis $\left({ }^{\mathrm{o}}\right.$ Brix$)$ & $67,4^{\mathrm{ab}} \pm 0.35$ & $66,5^{\mathrm{ab}} \pm 0.21$ & \\
\hline
\end{tabular}

* Médias seguidas de letras diferentes numa mesma linha indicam que houve diferença estatística significativa com probabilidade de erro (p $\leq 5 \%$ ) pelo teste Tukey. F1(34,8\% de abacaxi e de coco) F2 (coco verde 50\%, abacaxi 24,25\%) e F3 (coco verde 59,7 e abacaxi $10 \%$ ). Fonte: Autores (2020).

A umidade apresentou-se mais elevada na medida em que se aumentou a proporção de coco verde nas formulações. Diversos estudos evidenciaram que o albúmen sólido do coco verde possui umidade elevada a exemplo de Machado et al., (2020) que encontraram 90,2\% de umidade e Leal et al., (2013) que encontraram 87,1\% de umidade. Nos doces, além da evaporação de parte da água no processo de cozimento, a adição da sacarose diminui a disponibilidade de água na formulação final.

Em relação ao $\mathrm{pH}$, todas as formulações apresentaram-se como ácidas sendo observado que os valores de $\mathrm{pH}$ aumentaram e a acidez diminuiu na medida em que se aumentou a quantidade de coco e diminuiu-se a quantidade de abacaxi, sendo possivelmente o abacaxi o responsável pela maior acidez. Cabe destacar que na literatura estão disponíveis dados do pH do albúmen sólido do coco verde e que este é próximo à neutralidade conforme evidenciado por Teixeira et al., (2019) que 
obteve valores de 6,3 e Leal et al., (2013) que obteve pH de 6,7 no albúmen sólido do coco verde. Na F3, formulação com maior quantidade de coco verde o pH fica próximo aos valores supracitados $(6,02)$ e na F1 que é a formulação com menor quantidade de coco o pH é mais ácido 5,01.

Além de ser positivo em relação ao sabor, a diminuição do $\mathrm{pH}$ é interessante também por contribuir na ampliação da conservação dos produtos haja vista que em pH próximo a neutralidade o produto ficaria mais susceptível à multiplicação microbiana (Cereser et al., 2008, Kato et al., 2013).

Em relação aos valores de sólidos solúveis não houve diferença estatística entre as formulações ( $p>0,05)$ haja vista as quantidades de açúcar adicionadas terem sido próximas e compensadas pelos sólidos dos vegetais. Todas as formulações atendem ao recomendado pela legislação que determina que para doces cremosos o Brix seja superior a 55. (Brasil, 1978).

Torrezan et al., (2018) desenvolveram e caracterizaram cocadas de coco verde e frutas tropicais com uma das formulações levando 59,8\% de coco verde, $20 \%$ de açúcar e $20 \%$ de abacaxi, formulação semelhante a F3, e obteve valores semelhantes de $\mathrm{pH}$ e sólidos solúveis e valores de acidez superiores aos aqui obtido.

Os dados da avaliação sensorial estão apresentados na Tabela 3. Todas as formulações foram aceitas pelos painelistas haja vista terem médias acima do ponto neutro (5 - nem gostei/nem desgostei).

Tabela 3. Escores médios e desvios-padrão da avaliação sensorial dos doces cremosos produzidos com coco verde e abacaxi.

\begin{tabular}{lccc}
\hline \multirow{2}{*}{ Variáveis } & \multicolumn{3}{c}{ Formulações } \\
\cline { 2 - 4 } & $\mathbf{F 1}$ & $\mathbf{F 2}$ & $\mathbf{F 3}$ \\
\hline Aparência & $7.42^{\mathrm{a}} \pm 1.38$ & $7.05^{\mathrm{a}} \pm 1.28$ & $6.72^{\mathrm{a}} \pm 1.76$ \\
Cor & $7.51^{\mathrm{a}} \pm 1.40$ & $7.02^{\mathrm{ab}} \pm 1.41$ & $6.60^{\mathrm{b}} \pm 1.83$ \\
Aroma & $7.85^{\mathrm{a}} \pm 1.06$ & $7.58^{\mathrm{ab}} \pm 1.50$ & $7.05^{\mathrm{b}} \pm 1.73$ \\
Sabor & $7.69^{\mathrm{a}} \pm 1.32$ & $7.38^{\mathrm{ab}} \pm 1.67$ & $6.78^{\mathrm{b}} \pm 2.04$ \\
Textura & $7.65^{\mathrm{a}} \pm 1.47$ & $7.13^{\mathrm{ab}} \pm 1.63$ & $6.71^{\mathrm{b}} \pm 2.03$ \\
Avaliação Global & $7.72^{\mathrm{a}} \pm 1.22$ & $7.26^{\mathrm{ab}} \pm 1.38$ & $6.76^{\mathrm{b}} \pm 1.69$ \\
\hline
\end{tabular}

* Médias seguidas de letras diferentes numa mesma linha indicam que houve diferença estatística significativa com probabilidade de erro (p $\leq 5 \%$ ) pelo teste Tukey. F1(34,8\% de abacaxi e de coco) F2 (coco verde 50\%, abacaxi 24,25\%) e F3 (coco verde 59,7 e abacaxi $10 \%$ ). Fonte: Autores (2020).

Em se tratando do atributo aparência não houve diferença estatística significativa ( $\mathrm{P}>0,05)$. Para todos os demais atributos avaliados (cor, aroma, sabor, textura e avaliação global) houve diferença estatística significativa ( $\mathrm{P}<0,05)$, havendo diferenciação entre a F1 (quantidades iguais de coco e abacaxi) e a F3(maior percentual de coco verde adicionado) sendo que a F1 apresentou escores maiores em todos os atributos avaliados e a F3 os menores. Quando comparadas a F1 e a F2 (50\% de coco verde) não houve diferença estatística em nenhum dos atributos ( $p>0,05)$.

Os painelistas também foram convidados a avaliar as formulações de doce cremoso quanto a intenção de compra. Os resultados estão apresentados na Figura 1. 
Figura 1. Intenção de compra dos doces cremosos produzidos com coco verde e abacaxi.

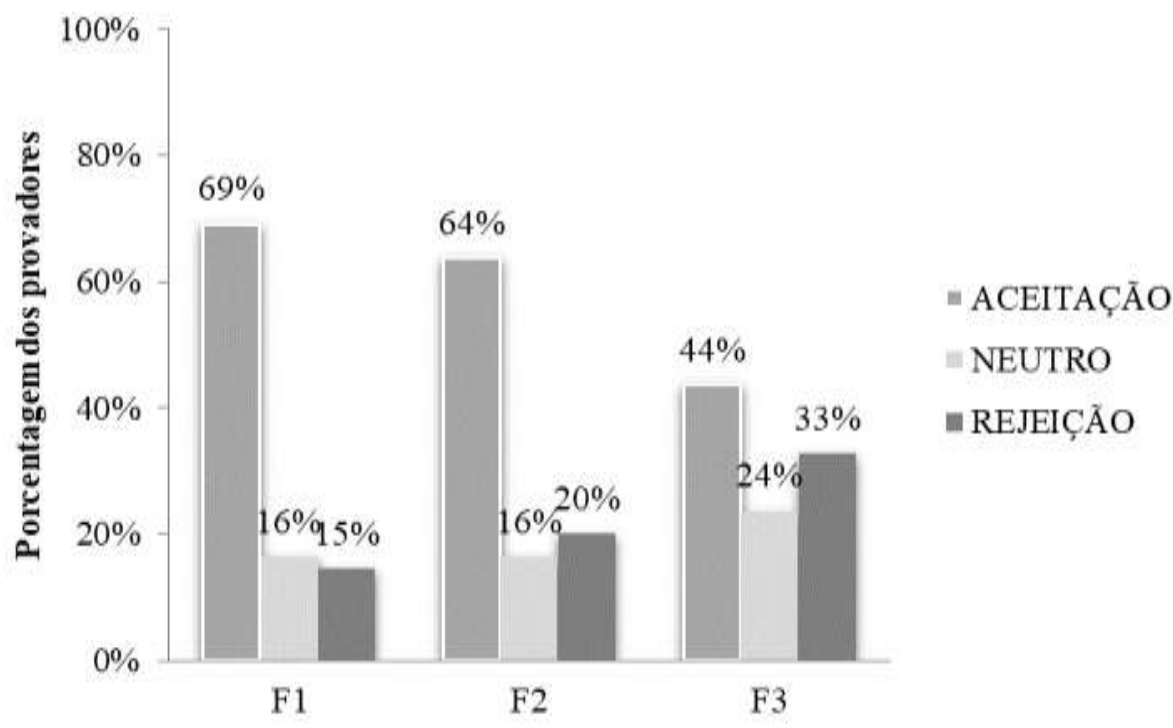

Formulações

F1(34,8\% de abacaxi e de coco) F2 (coco verde 50\%, abacaxi 24,25\%) e F3 (coco verde 59,7 e abacaxi 10\%). Fonte: Autores (2020).

Os painelistas tiveram os conceitos atribuídos as formulações no que tange a intenção de compra distribuídos em aceitação (quando atribuído conceito 4 -Possivelmente compraria ou 5-Certamente compraria) neutro (quando atribuído conceito 3 - talvez comprasse/talvez não comprasse) e rejeição (quando atribuído 2 - possivelmente não compraria ou 1certamente não compraria). Todas as formulações tiveram maior percentual de painelistas atribuindo conceitos ligados a intenção de comprar o doce caso encontrassem no mercado. No entanto, o mesmo comportamento da avaliação sensorial aparece na avaliação da intenção de compra: a formulação F1 (quantidades iguais de coco verde e abacaxi) apresenta o maior percentual de intenção de compra seguida da F2 e a formulação com 60\% de coco teve o maior percentual de rejeição (33\%) e foi a única que quando somados os que ficaram neutros e o percentual de rejeição o percentual supera a aceitação.

\section{Conclusão}

A utilização do albúmen sólido de coco verde apresentou-se como alternativa viável para utilização na produção de doce cremoso misto de coco e abacaxi haja vista ter resultado em produtos microbiologicamente seguros com características físico-químicas dentro do previsto pela legislação e bem aceitos sensorialmente. O desenvolvimento de doces utilizando polpa de coco verde é uma alternativa para combater o desperdício dessa matéria-prima de boa qualidade nutricional, além de ter o potencial de agregar valor econômico à cadeia do coco no Brasil. Para isto, a condução de novos estudos que explorem o potencial de utilização desta matéria-prima no desenvolvimento de novos produtos devem ser realizados.

\section{Referências}

AOAC. Association of Official Analytical Chemists. (2016) Official Methods of Analysis of AOAC International. (18a ed.), 3000p.

APHA. American Public Health Association. Committee on Microbiological for Foods. (2001). Compendium of methods for the microbiological examination offoods. (4a ed.), Washington:American Public Health Association, 676p.

Benassi, A. C., Ruggiero, C., Martins, A. B. G. \& Silva, J. A. (2007). Caracterização biométrica de frutos de coqueiro, Cocos nucifera L. variedade anã-verde, em diferentes estádios de desenvolvimento. Revista Brasileira de Fruticultura, 29(2), 302-7. 
Brainer, M. In: Brainer, M. (2018) Produção de coco: o Nordeste é destaque nacional. 61. Caderno Setorial Escritório Técnico de Estudos Econômicos do Nordeste ETENE, E-book.

Brasil. Ministério da Saúde. Agência Nacional de Vigilância Sanitária - ANVISA. (1978). Resolução Normativa $n^{\circ} 12$ de 24 de julho de 1978 . Regulamento técnico para padrões de identidade e qualidade de alimentos e bebidas. Diário Oficial [da] República Federativa do Brasil, Brasília.

Brasil. Ministério da Saúde. Agência Nacional de Vigilância Sanitária. (2019). Instrução normativa no 60, de 23 de Dezembro de 2019. Estabelece as listas de padrões microbiológicos para alimentos. Diário Oficial da República Federativa do Brasil, Brasília.

Cereser, N. D., Costa, F. M. R., Junior, O. D. R., Silva, D. A. R. \& Sperotto, V. R. (2008). Botulismo de origem alimentar. Ciência Rural, 38(1), 280-7. https://www.scielo.br/pdf/cr/v38n1/a49v38n1.pdf

Dias, L. F. (2019) Conservação do albúmen sólido do coco verde (Cocos nucifera L.) por tratamento térmico. Dissertação. Mestrado em Engenharia de Processos Químicos e Bioquímicos. Universidade Federal do Rio de Janeiro, 94p.

Gil, A. C. (2002). Como elaborar projetos de pesquisa. (4a ed.), Atlas.

IBGE. Instituto Brasileiro de Geografia e Estatística. (2017). Levantamento Sistemático da Produção Agrícola. Volume 30.

Kato, T., Ribeiro, K.P., Bordonal, V. C., Silva, M. B. R., Oliveira, A. F. \& Seibel, N. F. (2013) Avaliação da qualidade de doces de frutas agroindustriais do norte do Paraná. Revista Brasileira de Produtos Agroindustriais 15(2), 173-182. http://www.bibliotekevirtual.org/index.php/2013-02-07-03-02-35/2013-0207-03-03-11/1428-rbpa/v15n02/15283-avaliacao-da-qualidade-de-doces-de-frutas-agroindustriais-do-norte-do-parana.html

Leal, R. C., Barros, L. R., Mouchrek Filho, V. E., Mendes Filho, N. E., Everton, P. C. \& Luz, D. A. (2013) Estudo físico-químico da polpa de coco verde (Cocos nucifera L.) in natura, comercializado em praias de SÃO LUÍS-MA. In: Congresso Brasileiro de Química, Anais...

Lima, E. B. C., Sousa, C. N. S., Meneses, L. N., Ximenes, N. C., Santos-Junior, M. A. \& Vasconcelos, G. S. (2015). Cocos nucifera Linn (Arecaceae): a phytochemical and pharmacological review. Brazilian Journal of Medical and Biological Research, 48(11), 953-64. https://www.scielo.br/scielo.php?script=sci_arttext\&pid=S0100-879X2015001100953

Luz, D. S., Oliveira, M. V. S., Mouchrek, A. N., \& Bandeira, M. G. A. (2020). Elaboração, caracterização nutricional e microbiológica de iogurtes com adição de coco queimado e calda de coco, preparados a partir de polpa de coco verde da espécie (Cocus nucifera L.). Brazilian Journal of Development. 6(3), 1228395. 10.34117/bjdv6n3-187.

Machado, L. J., Sartori, R. A., Marques, D. D., Nascimento, A. E. S. \& Furtado, J. M. Utilização da biomassa do coco verde (Cocos nucifera L.) para obtenção de subprodutos. Brazilian Journal of Development. 6(1), 3808-26. 10.34117/bjdv6n1-270

Martins, C. R. \& Jesus Junior, L. A. (2014). Produção e Comercialização de Coco no Brasil Frente ao Comércio Internacional: Panorama 2014. Embrapa Tabuleiros Costeiros, Documentos $184,51 \mathrm{p}$.

Meilgaard, M., Civille, G. V., \& Carr, B.T. (2006). Sensory Evaluation Techniques. CRP Press, (4a ed.), Inc. 466p.

Silva, S. I. S (2019). Aproveitamento da biomassa de coco verde para produção de biocombustíveis sustentáveis. Dissertação. Mestrado em Desenvolvimento e Meio Ambiente. Universidade Federal da Paraíba

Silveira, M. A. G., Silveira, C. M., Cogo, S. L., Meira, S. M. M., Gautério, F. G. A. \& Santos, J. R. G. (2020). Desenvolvimento e caracterização de doce cremoso de bagaço de uva vinificada. Brazilian Journal of Development, 9(9), 1-17. doi.org/10.33448/rsd-v9i9.7222

Stone, H. \& Sidel, J. L. (2004). Sensory Evaluation Practices. (3a ed.). Academic Press, 408p.

Teixeira, N. S., Torrezan, R., De Grandi, D., Freitas-Sá, C., Pontes, S. M., Ribeiro, L. O., Cabral, L. M. C. \& Matta, V. M. (2019). Development of a fruit smoothie with solid albumen of green coconut. Ciência Rural, 49(1), 1-8. https://doi.org/10.1590/0103-8478cr20180110.

Torrezan, R., (2015). Doce em massa. EMBRAPA, 68p. (Coleção Agroindústria Familiar).

Torrezan, R., Pacheco, I. S., Silva, P.S., Freitas, S.C., \& Freitas-Sá, D. G. C. (2018) Aproveitamento do albúmen sólido de coco verde para a elaboração de cocadas adicionadas de frutas tropicais. In: XXXVI Congresso Brasileiro de Ciência e Tecnologia de Alimentos, Belém. Anais...

Vysakh, A., Ratheesh, M., Rajmohanan, T. P., Pramod, C., Premlal, S., Girish kumar, B. \& Sibi, P.I. (2014). Polyphenolics isolated from virgin coconut oil inhibits adjuvant induced arthritis in rats through antioxidant and anti-inflammatory action. International Immunopharmacology, 20(1), 124-30. https://doi.org/10.1016/j.intimp.2014.02.02 